\title{
Looking with a paralysed eye: adaptive plasticity of the vestibulo-ocular reflex
}

\author{
BRUNO ESTAÑOL, ${ }^{*}$ GUILLERMO LOPEZ-RIOS $\dagger$ \\ From the Departments of Neurology* and Neuro-otology, $\dagger$ Hospital General, Centro Medico Nacional, \\ Instituto Mexicano Del Seguro Social, Mexico City, Mexico
}

SUMMARY When a patient with a peripheral monocular paresis is forced to look with the paretic eye, head movements induce the sensation of an unstable visual world. The patient behaves as if he had acute bilateral labyrinthine lesions. These symptoms are due to the lack of compensatory ocular movement and the patients complain that the visual objects move in the direction opposite to the head. The patients develop ataxia, nausea, vomiting and past pointing. The symptoms, however, are transient and consistently disappear after approximately 48 hours. The central adaptation to looking and seeing with the paralysed eye is associated with a plastic change of the VOR. This plastic adaptation is probably induced by the large retinal slip produced by the lack of compensatory movement of the eye and can be studied in the normally mobile eye in the dark. The psychophysical adaptation is probably generated by an efferent copy or corollary discharge of the vestibular system to the visual system that cancels the retinal error.

When a patient with a monocular paresis is forced to use his paretic eye by patching his normally mobile eye, head movements induce the sensation of an unstable visual world. The subject develops ataxia, nausea and vomiting. He develops hypermetria in the finger to nose test with consistent over-shooting. If he lies still without moving the head, the sensation that the visual surroundings are moving decreases. The patient behaves as if he had acute bilateral labyrinthine lesions. Nevertheless, the symptoms consistently disappear after the sound eye is persistently covered for 24 to 48 hours. The patient is able to walk again without ataxia. The nausea and vomiting and the overshooting vanish. The patient states that the oscillopsia is greatly diminished. Since the eye is paralysed there has occurred a central compensation in subjects with a normal central nervous system. How does this compensation come about? Two types of adaptation may explain these phenomenon. A plastic change in the motor output of the vestibular system or the neurons of the oculomotor nuclei or a psycho-physical adaptation

Address for reprint requests: Dr Bruno Estañol, Division de Medicina, Hospital General, Centro Medico Nacional, Instituto Mexicano Del Seguro Social, Cuauhtemoc 330, Mexico 7, D.F., Mexico City Code: 06720.

Received 20 September 1983 and in revised form 4 February. 1984. Accepted 18 February 1984 to the increased retinal slip.

We postulated that the plastic adaptation of the vestibulo-ocular reflex (VOR) in turn, may be an increase in the gain of the reflex that attempts to compensate the large retinal error or a decrease in the gain in the reflex in order to diminish the mismatch in the brain between visual and vestibular impulses. An adaptive plasticity in the vestibuloocular responses to a change in the visual retinal error has been well demonstrated in the monkey and normal human subjects. ${ }^{3-6}$ Thus, an increase in retinal error by wearing $2 \times$ magnifying lenses, has been shown to induce a high gain of the VOR. We decided to investigate the behaviour of the gain of the VOR in the normally mobile eye in darkness in patients with monocular paralysis in order to elucidate the central mechanisms operating when a patient with a paretic eye is forced to look with the abnormal eye, the sound eye being covered. This model is ideal to study changes in gain of the VOR in human subjects because the normally mobile eye receives a normal efferent outflow of impulses from the vestibular and the ocular-motor systems.

\section{Patients and methods}

Patient 1

A 28-year-old female, with a history of migraine, developed a week prior to her admission, severe pain in the 


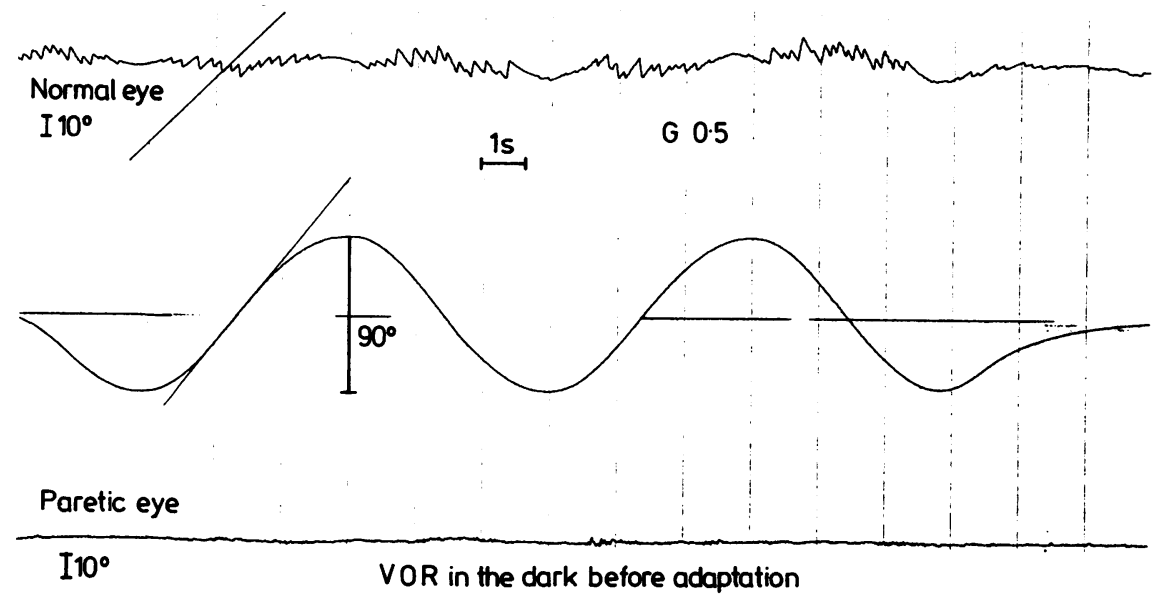

Fig 1 VOR in the dark before adaptation. Gain 0.5.

right orbital region. Four days after the onset of the pain she noticed double vision in all directions. Physical examination on the day of admission revealed: (1) Slight ptosis of the right side; (2) Decreased corneal reflex on that side; (3) Normal pupils; (4) The movements of the right eye were greatly diminished in all directions but particularly abduction and adduction. Vertical eye movements were restricted in all directions. An injection of $10 \mathrm{mg}$ of edrophomiun chloride did not alter the paresis. The erythrocyte sedimentation rate was $90 \mathrm{~mm}$ in one hour. The following studies were normal: CT scanning with contrast, an EEG, lumbar CSF, VDRL, FTA, immunoelectrophoresis and chest radiograph. A diagnosis of painful ophthalmoplegia of Tolosa-Hunt was made. She received prednisone $60 \mathrm{mg}$ a day for 15 days with gradual resolution of her symptoms.

\section{Patient 2}

A 39-year-old male was seen 25 days after the onset of double vision in all directions. He suddenly noticed pain in his left eye and 24 hours later he developed diplopia, palpebral ptosis and pain in the left side of the face. There were no systemic symptoms. Physical examination revealed $40 \%$ ptosis of the left eye, movements of the left eye paralysed in all directions, and a left sided peripheral facial palsy. CT scanning, lumbar CSF, EEG and a left carotid angiogram were all normal. ESR was $60 \mathrm{~mm}$ in one hour. The diagnosis was probable Tolosa-Hunt syndrome. Complete recovery of symptoms occurred after eight weeks of therapy with $60 \mathrm{mg}$ of prednisone daily.

The patients were studied under three different conditions.

(1) The paretic eye was covered and the patient fixated with the sound eye for 4 days.

(2) The normal eye was patched and the patients were forced to fixate with the paretic eye for a period of 4 days. (3) Both eyes were left uncovered and the patients attempted to fixate with both eyes during the same time period. During this period the gain of the VOR was tested daily with sinusoidal chair rotations at 0.05 and $0 \cdot 1 \mathrm{~Hz}$. The period of the rotation at $0.05 \mathrm{~Hz}$ was 20 seconds with a peak acceleration of $18 \% \mathrm{~s}^{2}$. The period of rotation at $0 \cdot 1 \mathrm{~Hz}$ was 10 seconds with a peak acceleration of $36 \% \mathrm{~s}^{2}$. Each eye was recorded separately with DC EOG and a careful calibration was performed before and after each session. At least ten complete rotations were given to each patient every time. Chair velocity and displacement were also recorded. The VOR gain was calculated under two different conditions: (a) in the dark with eyes open and performing simple arithmetic calculations, (b) with lights on attempting to fixate with the paretic eye (normal eye covered). The gain of the vestibulo-ocular reflex was measured in the normally mobile eye and calculated by the ratio: mean eye velocity/mean chair velocity and also by the ratio: total eye displacement/total chair displacement. Both patients had normal visual acuity in both eyes, were on no medication and had no history of vestibular disease. The head was restrained and bent forward 30 degrees.

\section{Results}

\section{CLINICAL FINDINGS}

After the normal eye was patched two patients immediately developed ataxia, nausea and vomiting. Any head movement would induce the sensation that the stationary surroundings were moving. Head movements induced the sensation that the visual objects moved in the direction opposite to the head. If the patient remained still this visual sensation diminished. When the patients attempted to walk they displayed gross ataxia with a tendency to fall in any direction. Turning was particularly difficult, and they would turn very slowly always using their hands for support. They showed clear and consistent hypermetria with both hands on the finger to nose test during this period. The patients did not receive 


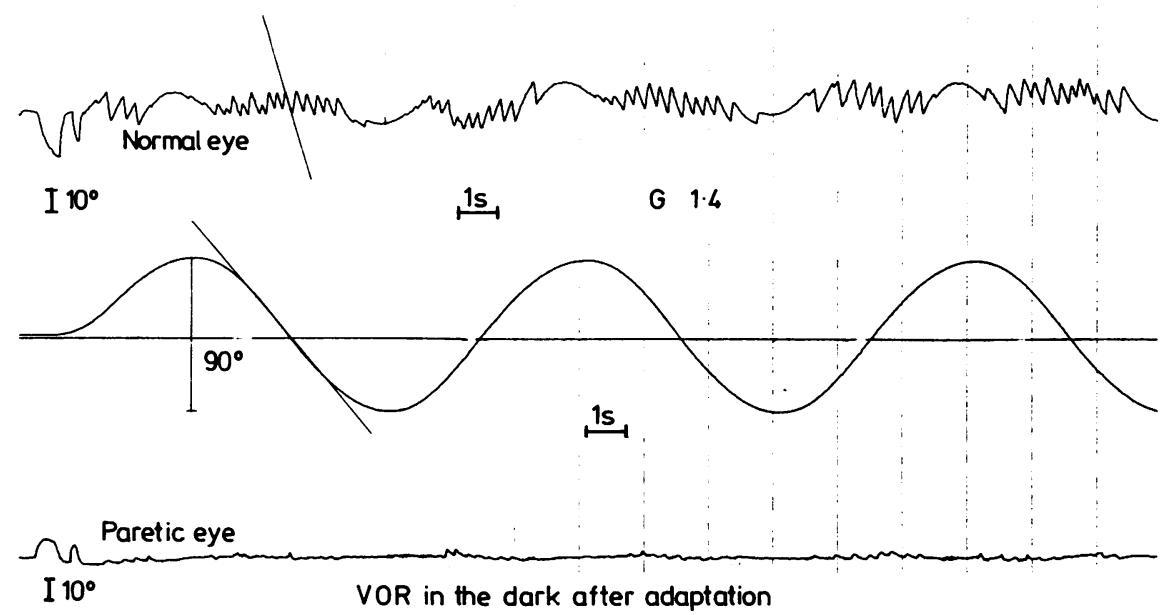

Fig 2 VOR in the dark after adaptation (after 72 hours of covering the normal eye). Gain 1.44.

medication (except prednisone) but received intravenous fluids because they could not tolerate oral liquids. After $\mathbf{4 8}$ hours both patients improved noticeably with disapppearance of the nausea and vomiting and were able to walk unaided. They claimed that the oscillopsia had almost vanished and could perform head movements and turns without much difficulty. After the normal eye was unpatched they developed again ataxia and past pointing but these symptoms were less intense and lasted less than 12 hours.
Gain of the VOR At the frequencies tested both patients had gains below 0.5 in the dark before adaptation The two patients had gains near one after 36 hours that they had been fixating with the paretic eye. After 72 hours, both patients had gains in the dark above 1 (figs 1,2 ). This difference is highly significant $(p<0.001)$. The gain of the VOR in the dark returned to the baseline 12 hours after the patients had the normal eye uncovered and used both eyes for fixation. The gains at 0.05 were lower than the gains at $0 \cdot 1 \mathrm{~Hz}$.

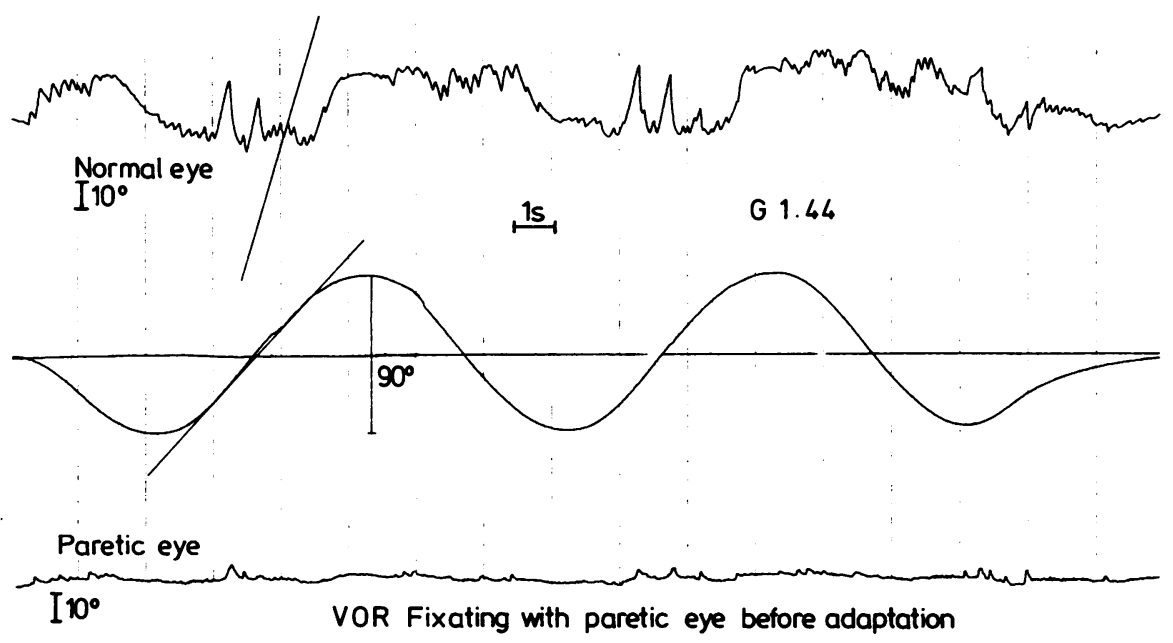

Fig 3 VOR while attempting fixation with the paretic eye with lights on, before adaptation. Gain 1.44. 


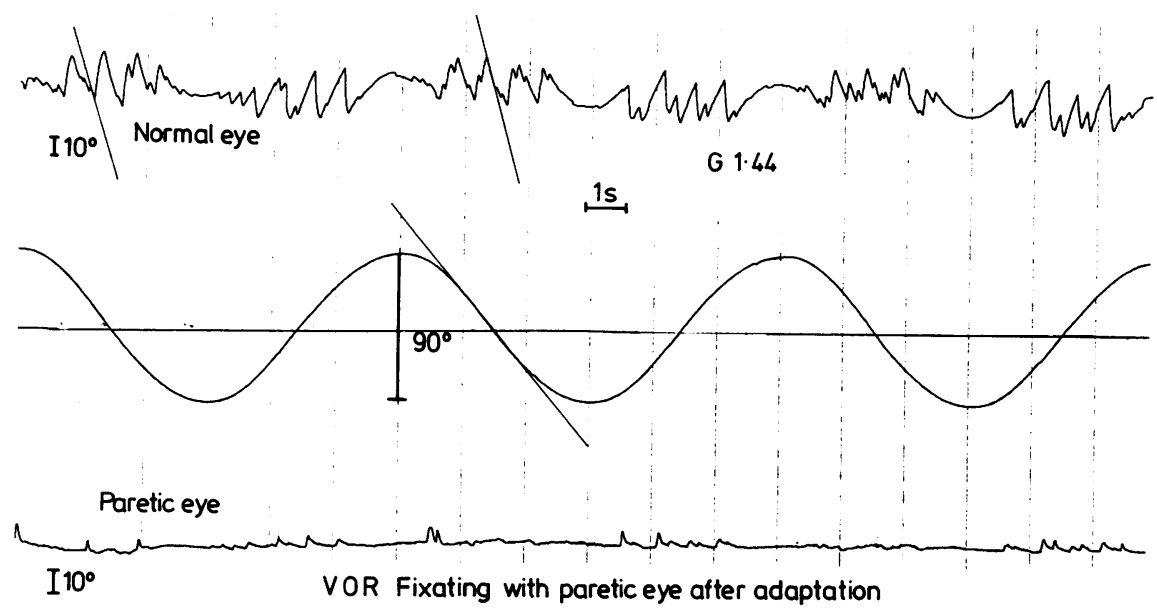

Fig 4 VOR while attempting fixation with the paretic eye with lights on after adaptation. Gain 1.44 .

$V O R$ in the dark before adaptation $0.05 \mathrm{~Hz}$ $0 \cdot 1 \mathrm{~Hz}$

VOR fixating with paretic eye before adaptation $1 \cdot 30$ $1 \cdot 45$

VOR in the dark after adaptation

VOR fixating with paretic eye after adaptation

The gains of the VOR while attempting fixation with the paretic eye were above unity and near 1.5 $($ at $0.1 \mathrm{~Hz})$ before and after adaptation. The gains were remarkably similar before and after adaptation (Graph 1) (figs 3,4). The gain in the dark after adaptation was, in both patients, equal to the gain fixating with the paretic eye with lights on.

\section{Discussion}

The function of the VOR is to maintain stable vision when the head moves by inducing compensatory eye movements in the opposite direction to the head. ${ }^{1613}$ The pursuit system keeps the image of a moving object in the macula. ${ }^{1}$ Patients who are forced to see solely with the paralysed eye do not have compensatory VOR on the paralysed side. Therefore, the lack of compensatory VOR produces an unstable visual world with head movements ${ }^{15}$ and the lack of pursuit blurrs vision of moving objects. An adaptive plasticity in the VOR has been induced by optical

means using magnifying or reducing telescopic spectacles altering the retinal image slip. ${ }^{2-6}$ Persistent retinal slip signals a need for a VOR adjustment of the gain. The gain in a paralysed eye is almost nil and the image of the stationary surrounding persistently moves in a direction opposite to that of the head movement.

The change in the VOR gain operates to improve stability of the retinal image during head turns; for this reason the system is said to have "adaptive gain control". The large retinal slip induced by the lack of compensatory movement due to the paralysis produces an increase in the gain of the VOR measured in the dark in the non-paralysed eye. How does this recalibration occur?

The large retinal slip induced by head rotation in light, fixating solely with a paralysed eye, stimulates the smooth pursuit system and the slow phase of OKN. The flocculus $P$ cells under these circumstances discharge in relationship to eye velocity ${ }^{10-12}$ and such impulses may be used as internal signals to calibrate the VOR. The "error signal" that calibrates the VOR may appear to be a combination of retinal slip and gaze velocity signal. On the other hand, patients fixating with a paralysed eye may obtain information from the "periphery" regarding eye velocity. If the eye is completely paralysed the retinal error velocity should be equal to the head velocity because the eye moves en bloc with the head.

If the eye is not totally paralysed, the retinal slip would be the difference between the eye and the head velocities. This information about head velocity may be used to supplement the head velocity 


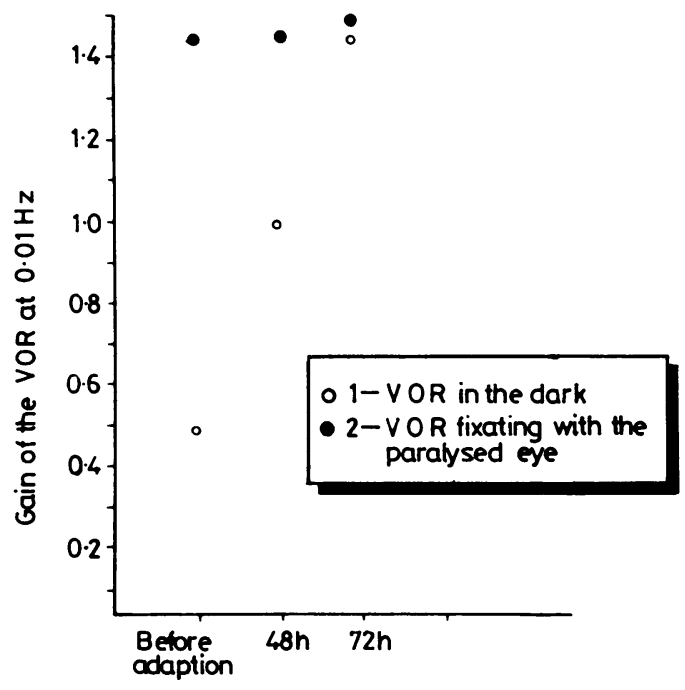

Fig 5 The VOR in the dark rose above the baseline after adaptation by a factor of two. This difference is highly significant $(p<0.001)$. The VOR while attempting fixation with the paretic eye with lights on, was the same before and after adaptation. The retinal slip velocity was the same before and after adaptation (see explanation in the text). (Mean data of the two patients.)

(a)

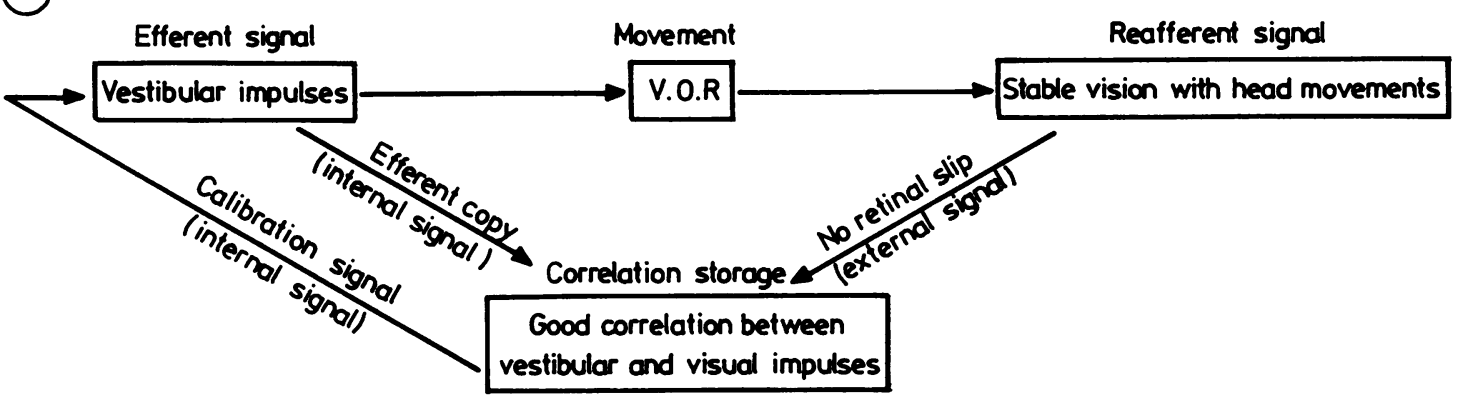

(b)

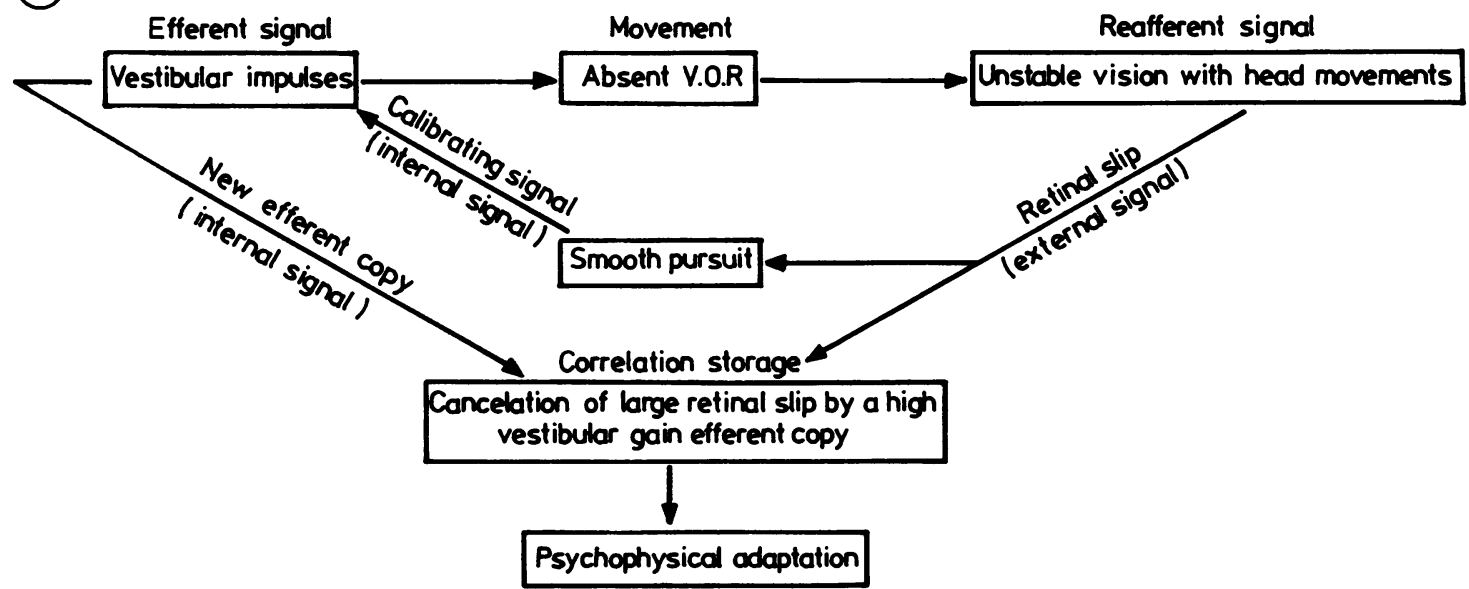

Fig 6 Reafference hypothesis. 
information given by the vestibular system. It appears that the retinal error velocity can exquisitely calibrate the VOR according to the amount of head velocity. The final calibration of the VOR is the result of the sum of the large retinal error velocity and the pre-existent VOR in the dark. Hence, the VOR gain in the dark increases to the exact point that can compensate the retinal error velocity signal and never above it. The VOR in the light while viewing with a paralysed eye may be considered as an "open loop" reflex because there is not correction "in line" by the visual system, and the increased gain under these circumstances may merely reflect the fact that all eye movements increase their gain under these conditions. ${ }^{16}$ The high gain may be considered simply as the consequence of the maximum open loop gain of which the VOR system is capable. Of course, the high gain during vestibular stimulation in the light when fixating with a paralysed eye increases the gain of the optokinetic system because this system acts normally under "closed loop" conditions," but it does not explain the persistent high gain of the VOR in the dark. The high gain of the VOR in the dark is an expression of plasticity because it persists despite the fact there is no visual input in the dark. The increased gain in the dark cannot be higher than the gain of the VOR in the light because the VOR has to be reset to a particular value of retinal error velocity which is also evidence of plasticity.

In addition to the increased gain of the VOR, a psychophysical adaptation occurs. This indicates that a balance between the expected and the actual retinal slip has occurred. The "reafference" hypothesis $^{7}$ postulates that the normal mechanism of movement consists of an efferent signal, an "efferent copy" of this signal, the movement and a reafferent signal caused by the movement. In this fashion the central nervous system can compare the intended with the actual movement. A mismatch between the reafferent impulses and the "efferent copy" of the movement would result in a recalibration of the outflow of the system. A "correlation storage" has been proposed that remembers previous combinations of efferent and reafferent signals. ${ }^{9}$ Using the "reafference" hypothesis we postulate that the correlation between visual and vestibular impulses takes place in a "correlation storage" where actual and expected retinal slips may be matched. If there is a great mismatch between visual and vestibular impulses, the activation of the $P$ cells of the flocculus of the pursuit system may serve as the internal signal that recalibrates the system, but it is difficult to conceive that it may also serve as the efferent copy of the expected retinal slip to be compared to the actual visual signal. In this schema it is therefore necessary to postulate an efference copy of the modified vestibular gain to induce the psychophysical adaptation (fig 6). In the experimental animal it has been demonstrated that visual cortical cells may be affected in their background or spontaneous firing rate by vestibular influences, ${ }^{17}$ emphasising the need of the visual signals to be compared to the vestibular signals. Recently, Wist et al $^{14}$ found that the subjective sensation of oscillopsia (and therefore, of retinal slip) decreases in patients with chronic monocular paresis. They suggest an "additional" mechanism that recalibrates the relationship between the expected and the actual retinal slip. They also considered the mismatch between actual and expected retinal slip to be the stimulus for this recalibration. The "adaptive suppression" of visual motion relieves the oscillopsia but may impair motion perception in general.

The authors wish to acknowledge the secretarial and editorial help of Mrs Emma Rosa Leroux.

\section{References}

' Robinson DA. Models of Oculomotor Neural Organization in the Control of Eye Movements. New York: Academic Press 1971:519-38.

${ }^{2}$ Miles FA, Fuller JH. Adaptive plasticity in the vestibulo-ocular responses of the rhesus monkey. Brain Res 1974;80:512-16.

${ }^{3}$ Gauthier GM, Robinson DA. Adaptation of the human vestibulo-ocular reflex to magnifying lenses. Brain Rev 1975;92:331-5.

${ }^{4}$ Gonshor A, Melvill Jones G. Plasticity in the adult human vestibulo-ocular reflex. Arch Proc Can Fed Biol Sol 1971;14:11.

${ }^{5}$ Miles FA, Eighmy BB. Long term adaptive changes in primate vestibulo-ocular reflex. I. Behavioral observations. J Neurophysiol 1980;43:1406-25.

- Miles FA, Disberger SG. Plasticity in the vestibuloocular reflex: A new hypothesis. Ann Rev Neurosci 1981;4:273-99.

7 Kornheuser AS. Adaptation to laterally displaced vision: A review. Psychol Bull 1976;83:783-816.

${ }^{8}$ Von Holst E. Relations between the central nervous system and the peripheral organs. British Journal of Animal Behaviour 1954;2:89-94.

${ }^{9}$ Held R. Plasticity in sensory-motor systems. Sci Am 1965;213:84-94.

${ }^{10}$ Miles FA, Braitman DS, Dow BM. Long-term adaptive changes in primate vestibulo-ocular reflex. IV. Electrophysiological observations in flocculus of adapted monkeys. J Neurophysiol 1980;43:1477-93.

" Miles FA, Fuller JH, Braitman DJ, Dow BM. Long-term adaptive changes in primate vestibulo-ocular reflex. III. Electrophysiological observations in flocculus of normal monkeys. J Neurophysiol 1980;43:1437-76.

12 Lisberger SG, Fuchs AF. Role of primate flocculus during rapid behavioral modification of vestibulo-ocular 
reflex. I. Purkinje cell activity during visually guided horizontal smooth-pursuit eye movements and passive head rotation. J Neurophysiol 1978;41:733-63.

${ }^{13}$ Ito M. Neural design of the cerebrellar motor control system. Brain Res 1971;40:81-4.

${ }^{14}$ Wist ER, Brandt TH, Krafczyk S. Oscillopsia and retinal slip. Brain 1983;106:153-68.

is Gresty MA, Hess K, Leech J. Disorders of the vestibulo-ocular reflex producing oscillopsia and mechanisms compensating for loss of labyrinthine function. Brain 1977;100:693-716.

${ }^{16}$ Kommerell G, Olivier G, Theopold H. Adaptive programming of phasic and tonic components in saccadic eye movements. Invest Ophthalmol 1976; 15:657-60.

${ }^{17}$ Lahue RH, Reinis S, Landolt JP, Money KE. VisualVestibular interactions in visual cortical cells in the cat. In: Cohen B, ed. Vestibular and Oculomotor Physiology. New York: New York Academy of Sciences. 1981:262-73. 